Conclusions Our findings extend the application of rosiglitazone by demonstrating that it significantly inhibits the LPS induced inflammatory response in RAW264.7 macrophages via PPAR $\gamma$ activation and NF- $\kappa$ B suppression.

\section{IDDF2019-ABS-0089 HSA_CIRC_0053063 ACTS AS A SPONGE OF MIR-361-3P IN COLON EPITHELIAL CELLS}

${ }^{1}$ Ying Shi*, 'Wei Huang, ${ }^{1}$ Shaohui Tang, ${ }^{2}$ Hongzhi $\mathrm{Xu},{ }^{2}$ Jianlin Ren. ${ }^{1}$ Jinan University, China; ${ }^{2}$ Xiamen University, China

\subsection{6/gutjnl-2019-IDDFabstracts.24}

Background The dynamic changes of serum circRNAs, which act as a sponge of microRNA, has a great potential in predicting colonic disease, especially in malignant diseases. However, whether the mechanism of circRNA in regulating colonic epithelial mucosa is still unknown. Here, we explore the sponging effects of hsa_circ_0053063 targeting miR-361-3p in colon epithelial cells.

Methods According to the ceRNA analysis, the potential target miRNAs of hsa_circ_0053063 were collected. Dual-luciferase reporter gene assays were performed to confirm the circRNA sponge of miRNAs. Then the potential target gene of miR361-3p was predicted. Dual-luciferase reporter gene assays and real-time PCR were performed to detect the regulation between ncRNAs and target gene in colon epithelial cells HCoEpiC and NCM460.

Results According to the ceRNA analysis, we found that miR361-3p might be a target of hsa_circ_0053063 (figure 1a). Dual-luciferase reporter gene assays demonstrated that hsa_circ_0053063 bound miR-361-3p directly (figure 1b). Then we predicted that ATG13 was a target gene of miR-361-3p. Dual-luciferase reporter gene assays demonstrated that miR361-3p bound ATG13 directly. Real-time PCR revealed that miR-361-3p decreased expression of ATG13, however, hsa_circ_0053063 reversed the inhibition of expression of ATG13 by $\mathrm{miR}-361-3 \mathrm{p}$.

Conclusions Hsa_circ_0053063 released the inhibition of expression of ATG13 by miR-361-3p. hsa_circ_0053063 may act as a sponge of miR-361-3p targeting ATG13 in colon epithelial cell.

IDDF2019-ABS-0090
CARBOXYLOMICS PROFILES DELINEATE
SHORT-CHAIN FATTY ACIDS IN
COLORECTAL CANCER DIAGNOSIS AND
PROGNOSIS

Jian-Lin Wu*, Xiqing Bian, Na Li. State Key Laboratory for Quality Research in Chinese Medicines, Macau University of Science and Technology, Macau

\subsection{6/gutjnl-2019-IDDFabstracts.25}

Background Colorectal cancer (CRC) is the third most common type of cancer in the world and is a major cause of worldwide cancer morbidity and mortality. Carboxylic acids widely exist in living systems and are the essential components for life, which mainly contain amino acids, TCA cycle intermediates, short-chain fatty acids, long chain fatty acids, bile acids, acylcarnitine, and so on. Carboxylomics study in biological samples is critical for the understanding of physiological processes and the discovery for the onset of relevant diseases. Methods In the present study, DIAAA derivatization-UHPLCQ-TOF/MS approach and caboxylomics study were employed to discover potential novel biomarkers for carboxylic acids in 58 human CRC and 46 healthy samples.

Results 269 carboxylic acids were determined and confirmed their structures. Among of them, 118 carboxylic acids were first reported in CRC serum. Metabolic pathways were constructed by heat maps, Interactive Pathways Explorer, pathway impact and Volcano plot, etc. (IDDF2019-ABS-0090 Figure 1. Heatmap of 134 carboxylic acids which have difference between healthy and CRC patients in training set (A); Interactive Pathways Explorer analysis (B); Metabolomic pathway of CRC samples in training set (C); Volcano plot of the 269 carboxylic acids profiled (D). Mann-Whitney $U$ tests were used to calculate statistical significance, and $\mathrm{p}$ values were corrected using Graphpad prism 5.0. Differentially abundant metabolites of different categories were individually color coded.) Shortchain fatty acids were found to be novel diagnostic and prognostic biomarkers for CRC (IDDF2019-ABS-0090 Figure 2. Relative abundance (A) and ROC curves (B) of representative a

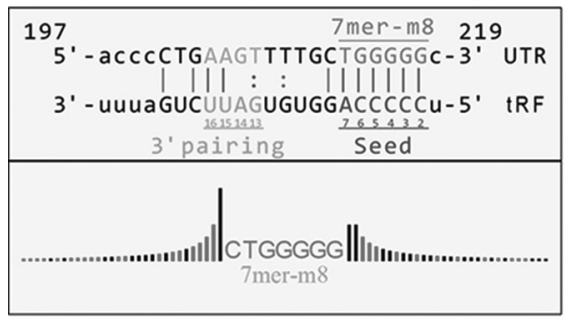

miR-control mimic miR-361-3p mimic miR-control inhibitor miR-361-3p inhibitor b

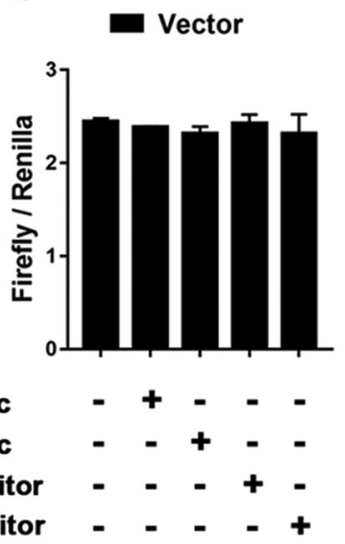

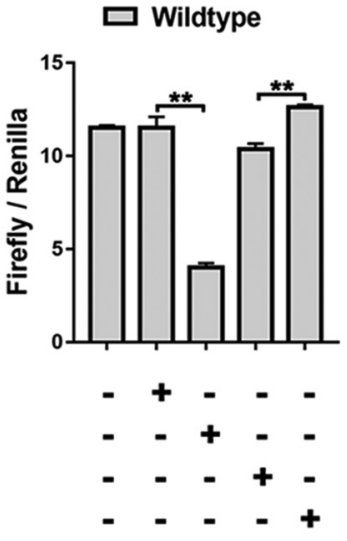

Abstract IDDF2019-ABS-0089 Figure 1 

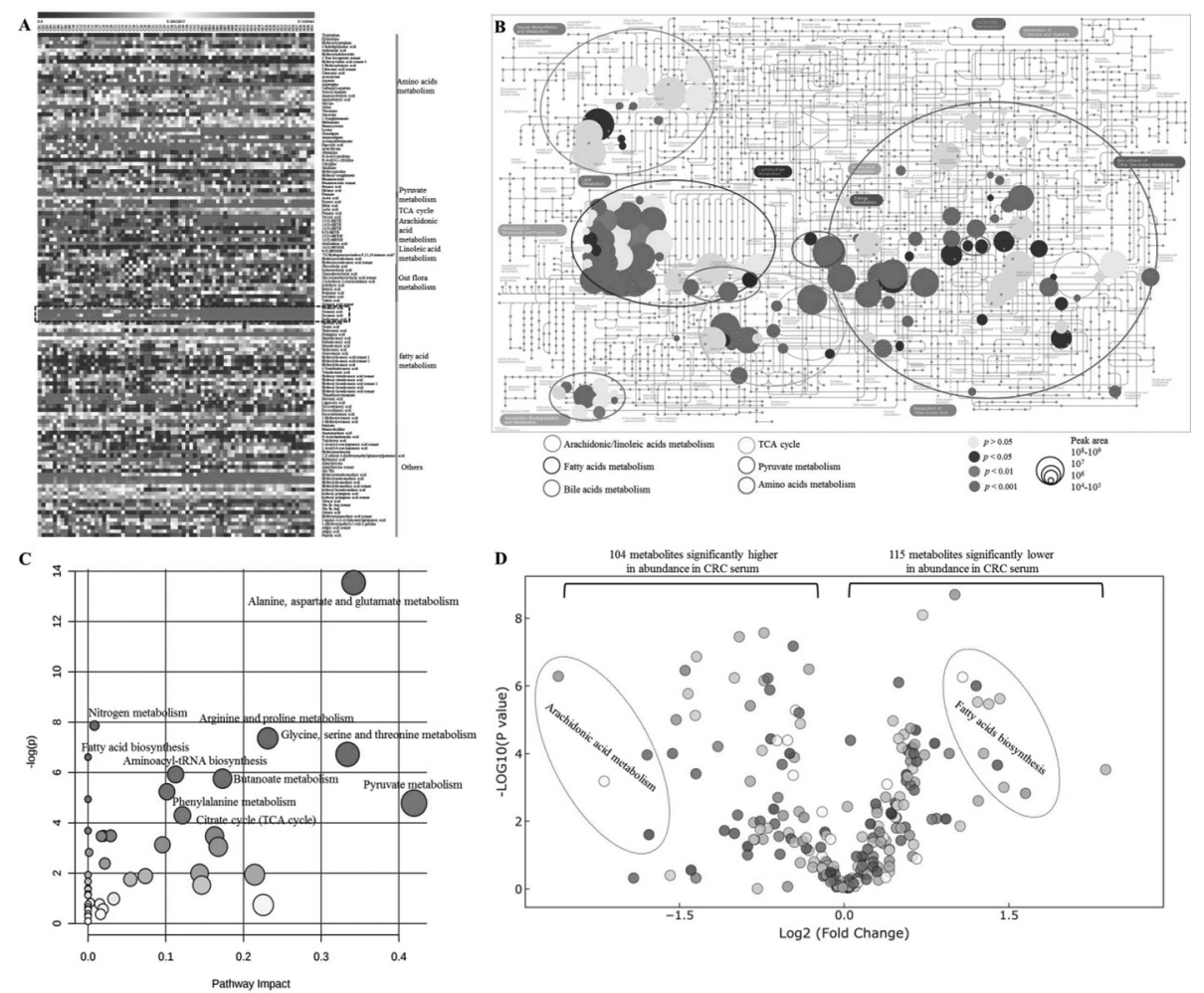

\section{Abstract IDDF2019-ABS-0090 Figure 1}
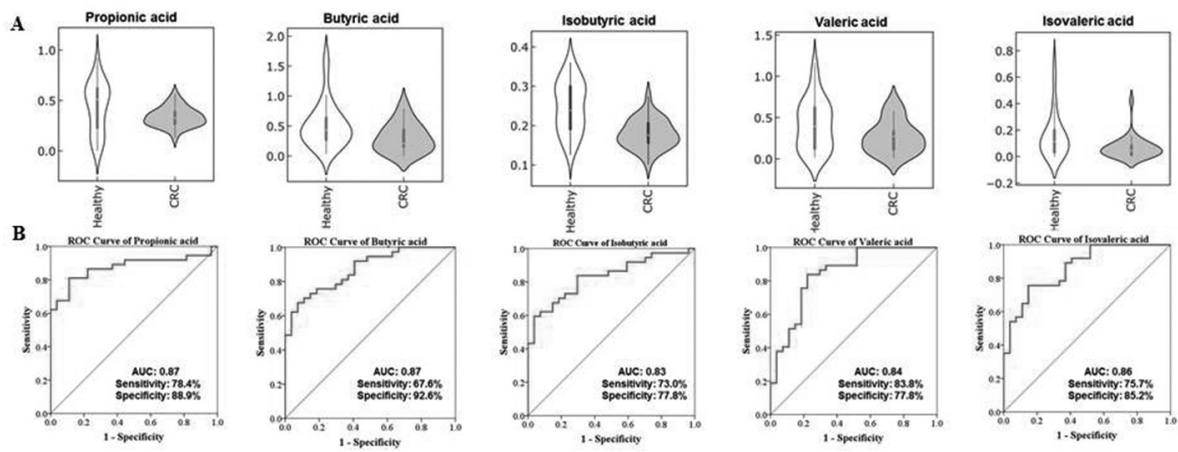

\section{Abstract IDDF2019-ABS-0090 Figure 2}

metabolites to differentiate CRC patients from healthy controls.).

Conclusions Overall, using DIAAA derivatization-UHPLC-QTOF/MS based carboxylomics study combined with network and ROC curve analysis, a new set of metabolites can be discovered as biomarkers of diseases with diagnostic and prognostic capabilities.

\section{IDDF2019-ABS-0106 EXOSOMES DERIVED FROM LEAN- COLORECTAL CANCER PATIENTS HAVE INCREASED IMMUNOSUPPRESSIVE EFFECTS ON CD8+T CELLS THAN OBESE- COLORECTAL CANCER PATIENTS}

Nadiah Abu*, Norahayu Othman, Nur Syahada Ab Razak, Siti Nurmi Nasir, Rahman Jamal. UKM Medical Molecular Biology Institute (UMBI), Malaysia
Background The role of obesity in relation to cancer, particularly colorectal cancer (CRC) is still an enigma. Although the underlying inflammation in obesity may contribute to poor prognosis, little is known on how this affects the efficacy of the immune system. Exosomes are extracellular vesicles that function as messengers between different cells. We postulate that exosomes derived from obese cancer patients are likely to deliver immunosuppressive signals towards the immune system, thus reducing the anti-tumor immunity.

Methods We isolated exosomes from the serum of 4 sets of patients, obeseCRC $(n=13)$, leanCRC $(n=15)$, obesenonCRC $(n=15)$ and leannonCRC $(n=15)$. The exosomes isolated were characterized via immunoblotting, electron microscopy and DLS methods. We further isolated fresh PBMCs from healthy volunteers $(n=4)$ and isolated CD8T cells using magnetic-bead based technique. Afterwards, the CD8T cells were activated and co-incubated with each of the isolated exosomes. Then, the apoptosis effects on CD8T cells were analyzed by annexin $\mathrm{V}$ assay, and the profile of related cytokines were determined 